\title{
Evolution of correlation between Helicobacter pylori infection and autoimmune liver disease
}

\author{
XIN-GUO PENG ${ }^{1}$, YU-YUAN LI ${ }^{2}$, HUI-TING CHEN ${ }^{2}$, YAN ZHOU $^{3}$, JIAN-GUO MA $^{4}$ and HONG-MIN YIN ${ }^{4}$ \\ ${ }^{1}$ Clinical Laboratory, The Affiliated Hospital of Binzhou Medical College, Binzhou, Shandong 256603; \\ ${ }^{2}$ Department of Gastroenterology, Guangzhou First People's Hospital, Guangzhou, Guangdong 510000; \\ ${ }^{3}$ Department of General Surgery, Yantai Mountain Hospital, Yantai, Shandong 264000; ${ }^{4}$ Department \\ of Gastroenterology, Binzhou Central Hospital, Binzhou, Shandong 256603, P.R. China
}

Received March 14, 2017; Accepted June 21, 2017

DOI: $10.3892 /$ etm.2017.4696

\begin{abstract}
The present study was planned to evaluate correlation between Helicobacter pylori (HP) infection and autoimmune liver disease (AILD). A total of 60 patients diagnosed with AILD in Affiliated Hospital of Binzhou Medical College were continuously enrolled in the present study. HP infection was detected by $13 \mathrm{C}$-urea breath test. The levels of anti-myeloperoxidase were tested by ELISA. The positive rate of anti-nuclear antibody (ANA), anti-mitochondrial antibody (AMA), anti-smooth muscle antibody (SMA) and anti-neutrophil cytoplasm antibody (ANCA) were tested by indirect immunofluorescence. The positive rates of anti-mitochondrial antibody (AMA-M2), anti-liver-kidney microsomal antibody (LKM-1), anti-liver cytoplasm antibody I (LC-1) and anti-soluble liver antigen/liver-pancreas antigen (SLA/LP) were tested by immunoblotting. Liver function indexes including alanine transaminase, aspartate transaminase, alkaline phosphatase and glutamyltransferase, were analyzed with a fully automatic biochemical analyzer. The levels of serum cytokine IFN- $\gamma$, interleukin-6 (IL-6), IL-10 and tumor necrosis factor- $\alpha$ (TNF- $\alpha$ ) were tested by ELISA. A total of 37 patients $(61.67 \%)$ were observed to be HP-positive. MPO-positive rate, positive rate of ANA, AMA, SMA and ANCA and positive rate of AMA-M2, LKM-1, LC-1 and SLA/LP in patients with positive HP infection were significantly higher than those of patients with negative HP infection. On the other hand, the levels of liver function indices did not showed any significant differences among HP-positive cases or HP-negative cases. However, the levels of IFN- $\gamma$, IL-6, IL-10 and TNF- $\alpha$ in patients with positive HP infection were significantly higher than those of patients with
\end{abstract}

Correspondence to: Dr Hong-Min Yin, Department of Gastroenterology, Binzhou Central Hospital, 108 South Huancheng Road, Binzhou, Shandong 256603, P.R. China

E-mail: hda5us@163.com

Key words: Helicobacter pylori, autoimmune liver disease, liver function, cytokines negative HP infection. In conclusion, the positive infection rate of HP infection in patients with AILD is high and is closely associated with various positive immune antibodies as well as cytokine levels.

\section{Introduction}

Autoimmune liver disease (AILD) is mainly caused by excessive activation of body immunity against own cells. This auto-activation of immunity further leads to a series of chronic and progressive liver diseases (1) like liver inflammatory response, liver cell and bile duct necrosis. AILD is mainly classified as primary biliary cirrhosis (PBC), autoimmune hepatitis (AIH) and primary sclerosing cholangitis (PSC). All three types of AILD are closely related to microorganism infection, biological heterologous materials, malnutrition, vaccination, ultraviolet radiation and environmental factors (2). Further, evolving advances in the fields of biochemistry, immunology and histopathology allowed efficient as well as specific detection of AILD, thus contributing towards timely management of AILD.

Helicobacter pylori (HP) is a Gram-negative bacilli planting in stomach and duodenum. HP infection is a major factor responsible for peptic ulcers (3). Moreover, HP could also exist in liver or kidneys. Moreover, there is a fair chance of its association with incidence of AILD (4). This study aims at analyzing the correlations between HP infection and AILD and will provide a new idea for clinical diagnosis and treatment of AILD.

\section{Patients and methods}

Study subjects. A total of 60 patients diagnosed with AILD (from January 2013 to January 2016 in Binzhou Hospital) were enrolled as study subjects in the present study. PBC diagnosis was performed in reference to the standards of American Association for the Study of Liver Diseases (AASLD, 2009). AIH diagnosis was referred to AASLD standard 2010; and PSC diagnosis was according to Mayer standards. AILD, liver cancer, viral hepatitis, alcoholic hepatitis, atrophic gastritis and gastric ulcers and pregnant/lactating women were excluded from the study. Study subjects included 39 male patients and 
Table I. Comparison of positive rate of ANA, AMA, SMA and ANCA (case, \%).

\begin{tabular}{|c|c|c|c|c|c|}
\hline Groups & $\begin{array}{c}\text { Case } \\
\text { no. }\end{array}$ & ANA & AMA & SMA & ANCA \\
\hline $\begin{array}{l}\text { HP-positive } \\
\text { infection }\end{array}$ & 37 & $18(48.65)$ & $16(43.24)$ & $15(40.54)$ & $20(54.05)$ \\
\hline $\begin{array}{l}\text { Negative } \\
\text { infection }\end{array}$ & 23 & $4(17.39)$ & $3(13.04)$ & $3(13.04)$ & $5(21.74)$ \\
\hline$\chi^{2}$ test & & 5.967 & 5.978 & 5.107 & 6.094 \\
\hline P-value & & 0.015 & 0.014 & 0.024 & 0.014 \\
\hline
\end{tabular}

ANA, anti-nuclear antibody; AMA, anti-mitochondrial antibody; SMA, antismooth muscle antibody; ANCA, anti-neutrophil cytoplasm antibody.

Table II. Comparison of positive rate of AMA-M2, LKM-1, LC-1 and SLA/LP (case, \%).

\begin{tabular}{|c|c|c|c|c|c|}
\hline Groups & $\begin{array}{c}\text { Case } \\
\text { no. }\end{array}$ & AMA-M2 & LKM-1 & LC-1 & SLA/LP \\
\hline $\begin{array}{l}\text { HP-positive } \\
\text { infection }\end{array}$ & 37 & $21(56.76)$ & $20(54.05)$ & $23(62.16)$ & $22(59.46)$ \\
\hline $\begin{array}{l}\text { Negative } \\
\text { infection }\end{array}$ & 23 & $2(8.70)$ & $2(8.70)$ & $3(13.04)$ & $3(13.04)$ \\
\hline$\chi^{2}$ test & & 13.859 & 12.566 & 13.935 & 12.572 \\
\hline P-value & & $<0.001$ & $<0.001$ & $<0.001$ & $<0.001$ \\
\hline
\end{tabular}

AMA-M2, anti-mitochondrial antibody II; LKM-1, anti-liver-kidney microsomal antibody I; LC-1, anti-liver cytoplasm antibody I; SLA/LP, antisoluble liver antigen/liver-pancreas antigen.

21 female patients, with age range of 45-78 years and mean age of $58.7 \pm 13.6$ years. This study was approved by the Ethics Committee of Binzhou Central Hospital. Signed written informed consents were obtained from the patients and/or guardians.

Study methods. HP infection was detected by 13C-urea breath test (13C-UBT). The levels of anti-myeloperoxidase (MPO) were tested by enzyme-linked immunosorbent assay (ELISA). The positive rate of rabbit polyclonal anti-nuclear antibody (ANA) (dilution, 1:100; cat. no. ab151216), rabbit polyclonal anti-mitochondrial antibody (AMA) (dilution: 1/100; cat. no. ab194396), rabbit monoclonal anti-smooth muscle anti-body (SMA) (dilution, 1:100; cat. no. ab108531) and rabbit polyclonal anti-neutrophil cytoplasm antibody (ANCA) (dilution, 1:500; cat. no. ab178623) were tested by indirect immunofluorescence (IIF). The positive rates of rabbit polyclonal anti-mitochondrial antibody (AMA-M2) (dilution, 1:500; cat. no. ab193668), rabbit monoclonal anti-liver-kidney microsomal antibody (LKM-1) (dilution, 1:500; cat. no. ab14554), rabbit polyclonal anti-liver cytoplasm antibody I (LC-1) (dilution, 1:500; cat. no. ab48394) and rabbit polyclonal anti-soluble liver antigen/liver-pancreas antigen (SLA/LP) (dilution, 1:500; cat. no. ab174136) were tested by immunoblotting (IBT). All antibodies were all purchased from Abcam (Cambridge, MA, USA). Liver function indexes
Table III. Comparison of liver function indexes (U/1).

\begin{tabular}{lcccc}
\hline Groups & ALT & AST & ALP & GGT \\
\hline $\begin{array}{l}\text { HP-positive } \\
\text { infection }\end{array}$ & $58.73 \pm 9.62$ & $53.84 \pm 14.52$ & $123.62 \pm 25.47$ & $49.68 \pm 15.05$ \\
Negative & $54.62 \pm 12.33$ & $51.22 \pm 13.65$ & $118.47 \pm 28.63$ & $47.52 \pm 14.66$ \\
infection & & & & \\
t-test & 0.162 & 0.184 & 0.231 & 0.206 \\
P-value & 0.965 & 0.932 & 0.857 & 0.883 \\
\hline $\begin{array}{l}\text { ALT, alanine transaminase; AST, aspartate transaminase; ALP, alkaline phos- } \\
\text { phatase; GGT, glutamyltransferase. }\end{array}$ &
\end{tabular}

Table IV. Comparison of IFN- $\gamma$, IL-6, IL-10 and TNF- $\alpha$ level $(\mu \mathrm{mol} / 1)$.

\begin{tabular}{lcccc}
\hline Groups & IFN- $\gamma$ & IL-6 & IL-10 & TNF- $\alpha$ \\
\hline $\begin{array}{l}\text { HP-positive } \\
\text { infection }\end{array}$ & $17.83 \pm 5.46$ & $45.62 \pm 12.23$ & $35.92 \pm 7.62$ & $13.68 \pm 4.25$ \\
Negative & $8.25 \pm 2.26$ & $24.35 \pm 10.24$ & $17.52 \pm 8.34$ & $6.56 \pm 2.73$ \\
infection & & & & \\
t-test & 8.624 & 7.965 & 9.234 & 9.625 \\
P-value & $<0.001$ & $<0.001$ & $<0.001$ & $<0.001$ \\
\hline
\end{tabular}

IL-6, interleukin-6; TNF- $\alpha$, tumor necrosis factor- $\alpha$.

alanine transaminase (ALT), aspartate transaminase (AST), alkaline phosphatase (ALP) and glutamyltransferase (GGT), were analyzed with a fully automatic biochemical analyzer. The levels of serum cytokine IFN- $\gamma$, interleukin-6 (IL-6), IL-10 and tumor necrosis factor- $\alpha(\mathrm{TNF}-\alpha)$ were tested by ELISA.

Statistical analysis. SPSS 20.0 (SPSS, Inc., Chicago, IL, USA) statistical software was used for statistical analysis. The measurement data were expressed as mean \pm standard deviation (SD) and comparison among groups adopted the independent sample t-test. Enumeration data were expressed by case number or (\%) and comparison among groups adopted $\chi^{2}$ test. $\mathrm{P}<0.05$ was considered to indicate a statistically significant difference.

\section{Results}

HP-positive rate analysis. A total of 37 patients (61.67\%) were observed to be HP-positive. HP ratio in positive patients was 4.1-7.6, with an average of 5.8 \pm 1.3 .

Comparison of MPO levels. The MPO-positive rate in HP-positive patients was significantly higher than that of HP-negative patients [35.14\% (13/37) vs. 8.70\% (2/23), $\left.\chi^{2}=5.288, \mathrm{P}=0.021\right]$.

Comparison of positive rate of ANA, AMA, SMA and ANCA. The positive rates of ANA, AMA, SMA and ANCA were observed to be significantly higher in HP-positive patients than that of HP-negative patients $(\mathrm{P}<0.05)$ (Table I). 
Comparison of positive rate of AMA-M2, LKM-1, LC-1 and SLA/LP. The positive rates of AMA-M2, LKM-1, LC-1 and SLA/LP were significantly higher in HP-positive patients in comparison to HP-negative patients $(\mathrm{P}<0.05)$ (Table II).

Comparison of liver function indexes. The levels of ALT, AST, ALP and GGT in HP-positive patients did not show any significant difference (Table III).

Comparison of IFN- $\gamma, I L-6, I L-10$ and TNF- $\alpha$ level. The levels of IFN- $\gamma$, IL-6, IL-10 and TNF- $\alpha$ in HP-positive patients were significantly higher than that of HP-negative patients $(\mathrm{P}<0.05)$ (Table IV).

\section{Discussion}

AILD could be preliminarily diagnosed by analyses of liver function tests. Significant elevation of ALT and AST enzyme activities confirm serious liver damage. However, conventional indexes are hard to identify between AILD and viral hepatitis (5). The mechanism of immune tolerance is missing in patients with AILD, thereby, inducing T lymphocyte-mediated damage.

High sensitive and specific antibodies included ANA, AMA, SMA, ANCA, MPO, AMA-M2, LKM-1, LC-1 and SLA/LP. ANA has been positively correlated with range of motion of diseases and responds to autoantibodies leading to inflammation and aggravation of the liver tissue damage in patients with AILD $(6,7)$. AMA is an autoantibody without organ specificity and observation of its higher levels signified necrosis, apoptosis or lymphoid infiltration into the intra-hepatic bile ducts (8). AMA-M2 is an ingredient of AMA, plays a leading role in AMA reaction and directly reflects the activeness of AMA (9). On the other hand, SMA is classified as one of diagnostic criteria in AILD guideline of AASLD, with species and organ-specific characteristics. The detection rate has been observed to be $70 \%$ in patients with AILD (10). When neutrophils receive antigenic stimulation in patients with AILD, the cytoplasm could produce ANCA that is associated with AILD activities and damage to the liver tissues (11). Further, MPO may inhibit the activation of neutrophils and inactivate the chemokines. However, in combination with ACNA, its inhibitory function was limited resulting in a large number of neutrophils leading to liver cell damage (12). Although the positive rates of anti-LKM-1, LC-1 and SLA/LP AILD are relatively low in the antigen spectrum, it is almost $100 \%$ in prediction of specificity. It is rather significant in diagnosis of AILD $(13,14)$.

The present study observed HP-positive rate of $61.67 \%$ in patients with AILD. HP contains some cytotoxin-related proteins (CagA). When HP is observed to be positive in patients with AILD, its concentration may be increasing and plenty of CagA may have transferred to the epithelial cells of gastric mucosa accordingly. The signaling pathway downstream of cells is thus activated resulting in aggravation of infection symptoms, thereby, inducing cellular immune response (15). When an AILD patients is in certain condition of immune tolerance, his/her defense capability would be reduced. Therefore, it may easily lead to endotoxemia resulting in generation of hepatic inflammatory injuries (16). Further in the present study MPO-positive rate, positive rates of ANA, AMA, SMA and ANCA, positive rates of AMA-M2,
LKM-1, LC-1 and SLA/LP were higher than in patients with negative HP. After HP infection, ANA has been reported to react with autoantibodies to form immune complexes resulting in immune dysfunction. Further, upon release of plasma inflammatory mediators in quantity, the inflammation has been reported to further aggravate the damage to liver tissues leading to AILD as observed in our study $(17,18)$. Moreover, observed elevation in MPO in combination with AMA and ANCA could be related to aggravation in the liver injury thereby causing lymphocytes to infiltrate into intra-hepatic bile duct and speed up liver cirrhosis $(19,20)$. Furthermore, the levels of IFN- $\gamma$, IL-6, IL-10 and TNF- $\alpha$ were significantly higher than in patients with negative HP. It suggested that the positive rate of HP infection in patients with AILD was rather high and was closely associated with elevation in various positive immune antibodies and cytokines. The present study concludes from the above observation that the ratio of correct diagnosis and clinical treatment outcome for AILD could be enhanced via early HP detection and intervention. However, the above results still need further confirmation in clinical setting with large sample size for a concrete conclusion.

\section{References}

1. Montano-Loza AJ, Thandassery RB and Czaja AJ: Targeting hepatic fibrosis in autoimmune hepatitis. Dig Dis Sci 61: 3118-3139, 2016.

2. Czaja AJ: Nature and implications of oxidative and nitrosative stresses in autoimmune hepatitis. Dig Dis Sci 13: 122-123, 2016.

3. Yeo SH and Yang $\mathrm{CH}$ : Peptic ulcer disease associated with Helicobacter pylori infection. Korean J Gastroenterol 67: 289-299, 2016 (In Korean).

4. El-Matary W, Dalzell AM and Ashworth M: Helicobacter pylori and autoimmune hepatitis. Eur J Pediatr 164: 54-55, 2005.

5. Muratori P, Lalanne C, Bianchi G, Lenzi M and Muratori L: Predictive factors of poor response to therapy in autoimmune hepatitis. Dig Liver Dis 48: 1078-1081, 2016.

6. Utiyama SR, Zenatti KB, Nóbrega HA, Soares JZ, Skare TL, Matsubara C, Muzzilo DA and Nisihara RM: Rheumatic disease autoantibodies in autoimmune liver diseases. Immunol Invest 45: 566-573, 2016.

7. Muratori L, Deleonardi G, Lalanne C, Barbato E, Tovoli A, Libra A, Lenzi M, Cassani F and Muratori P: Autoantibodies in autoimmune hepatitis. Dig Dis 33 (Suppl 2): 65-69, 2015.

8. Tomizawa M, Shinozaki F, Fugo K, Motoyoshi Y, Sugiyama T, Yamamoto S, Kishimoto T and Ishige N: Anti-mitochondrial M2 antibody-positive autoimmune hepatitis. Exp Ther Med 10: 1419-1422, 2015.

9. Sun L, Wang Y, Liu Y, Zhao Y, Zhang X, Wang Y, Zhao D, Zhang $\mathrm{H}$ and Ma Y: Differential characteristics of AMA-M2 autoantibody in primary biliary cirrhosis and non-PBC patients. Zhonghua Gan Zang Bing Za Zhi 23: 343-349, 2015 (In Chinese).

10. Longhi MS, Mieli-Vergani G and Vergani D: Autoimmune hepatitis. Curr Pediatr Rev 10: 268-274, 2014.

11. Stinton LM, Bentow C, Mahler M, Norman GL, Eksteen B, Mason AL, Kaplan GG, Lindkvist B, Hirschfield GM, Milkiewicz P, et al: PR3-ANCA: A promising biomarker in primary sclerosing cholangitis (PSC). PLoS One 9: e112877, 2014.

12. Nagai T, Imamura M, Kamiya Y and Mori M: Graves' disease accompanied by anti-myeloperoxidase antibody-related nephropathy and autoimmune hepatitis. Intern Med 43: 516-520, 2004.

13. Liberal R, Grant CR, Longhi MS, Mieli-Vergani G and Vergani D: Diagnostic criteria of autoimmune hepatitis. Autoimmun Rev 13: 435-440, 2014.

14. An IC, Tiwari AK, Ameda S and Laird-Fick HS: Autoimmune hepatitis: Diagnostic dilemma in the setting of suspected iron overload. Case Rep Gastrointest Med 2013: 872987, 2013.

15. Silva LD, Rocha AM, Rocha GA, de Moura SB, Rocha MM, Dani R, de Melo FF, Guerra JB, de Castr LP, Mendes GS, et al: The presence of Helicobacter pylori in the liver depends on the Th1, Th17 and Treg cytokine profile of the patient. Mem Inst Oswaldo Cruz 106: 748-754, 2011. 
16. Mackay IR, Leskovsek NV and Rose NR: Cell damage and autoimmunity: A critical appraisal. J Autoimmun 30: 5-11, 2008.

17. Dzierzanowska-Fangrat K, Nilsson I, Wozniak M, Jozwiak P, Rozynek E, Woynarowski M, Socha J, Ljungh A and Wadström T: Lack of an association between Helicobacter infection and autoimmune hepatitis in children. Pol J Microbiol 55: 157-159, 2006.

18. Vorobjova T, Nilsson I, Terjajev S, Granholm M, Lyyra M, Porkka T, Prükk T, Salupere R, Maaroos HI and Wadström T: Serum antibodies to enterohepatic Helicobacter spp. in patients with chronic liver diseases and in a population with high prevalence of $H$. pylori infection. Dig Liver Dis 38: 171-176, 2006.
19. Durazzo M, Pellicano R, Premoli A, Berrutti M, Leone N, Ponzetto A and Rizzetto M: Helicobacter pylori seroprevalence in patients with autoimmune hepatitis. Dig Dis Sci 47: 380-383, 2002.

20. Galperin C and Gershwin ME: Immunopathogenesis of gastrointestinal and hepatobiliary diseases. JAMA 278: 1946-1955, 1997. 